\title{
Screening and Functional Analysis of the Peroxiredoxin Specifically Expressed in Bursaphelenchus xylophilus-The Causative Agent of Pine Wilt Disease
}

\author{
Han-Yu Fu ${ }^{1}$, Jia-Hong Ren ${ }^{1,2}$, Lin Huang ${ }^{1}$, Hao $\mathrm{Li}^{1}{ }^{1}$ and Jian-Ren Ye ${ }^{1, *}$ \\ 1 College of Forest Resources and Environment, Nanjing Forestry University, Nanjing 210037, \\ Jiangsu, China; E-Mails: fuhanyu2019@gmail.com (H.-Y.F.); renjiahong04@gmail.com (J.-H.R.); \\ Lhuang@njfu.edu.cn (L.H.); lihao201404@gmail.com (H.L.) \\ 2 Department of Biological Science and Technology, Changzhi College, Changzhi 046011, \\ Shanxi, China
}

* Author to whom correspondence should be addressed; E-Mail: jryenjfu2014@gmail.com; Tel.: +86-139-0515-1921; Fax: +86-25-8542-7305.

Received: 21 April 2014; in revised form: 25 May 2014 / Accepted: 26 May 2014 /

Published: 10 June 2014

\begin{abstract}
The pine wood nematode, Bursaphelenchus xylophilus, is the causal agent of pine wilt disease. Accurately differentiating B. xylophilus from other nematodes species, especially its related species B. mucronatus, is important for pine wood nematode detection. Thus, we attempted to identify a specific protein in the pine wood nematode using proteomics technology. Here, we compared the proteomes of B. xylophilus and B. mucronatus using Two-dimensional gel electrophoresis (2-DE) and matrix-assisted laser desorption/ionization -time-of-flight/time-of-flight (MALDI-TOF/TOF-MS) technologies. In total, 15 highly expressed proteins were identified in B. xylophilus compared with B. mucronatus. Subsequently, the specificity of the proteins identified was confirmed by PCR using the genomic DNA of other nematode species. Finally, a gene encoding a specific protein (Bx-Prx) was obtained. This gene was cloned and expressed in E. coli. The in situ hybridisation pattern of Bx-Prx showed that it was expressed strongly in the tail of B. xylophilus. RNAi was used to assess the function of $B x-\operatorname{Prx}$, the results indicated that the gene was associated with the reproduction and pathogenicity of $B$. xylophilus. This discovery provides fundamental information for identifying B. xylophilus via a molecular approach. Moreover, the purified recombinant protein has potential as a candidate diagnostic antigen of pine wilt disease, which may lead to a new immunological detection method for the pine wood nematode.
\end{abstract}


Keywords: Bursaphelenchus xylophilus; comparative proteomics; 2-DE; in situ hybridization; prokaryotic expression

\section{Introduction}

The pine wood nematode (PWN), Bursaphelenchus xylophilus (Steiner \& Buhrer) Nickle, is the primary pathogen causing rapid wilting of Pinus thunbergii Parl. and P. densiflora Siebold \& Zucc. in East Asia and $P$. pinaster Aiton in Portugal Europe, resulting in the deaths of millions of pine trees annually [1-4]. Correctly differentiating B. xylophilus from other species of nematodes in pines is the key to controlling and preventing the rapid spread of pine wilt disease.

However, identification of B. xylophilus is complicated by Bursaphelenchus mucronatus Mamiya \& Enda [5]. B. mucronatus, a very similar species to B. xylophilus in morphology and biology, was discovered in the wood of dead pine trees and described as a new species [6]. It was reported that B. mucronatus has very low virulence or no pathogenicity to host pine trees compared with B. xylophilus [6-8]. Thus, differentiating these two species is crucial.

The traditional method of distinguishing these species is based on morphological differences. Mamiya and Enda (1979) [6] distinguished B. xylophilus from B. mucronatus according to its rounded tail shape with no distinct mucron. However, Wingfield et al. (1983) [9] found that female B. xylophilus from North America showed variations in tail shape from rounded to mucronated. Thus, identification of the two species using morphological characters alone may lead to misidentification [10-12]. Moreover, morphological detection is time- and labour-intensive.

Presently, serological techniques are the major means of detecting bacteria, viruses, and phytoplasmas and play a very important role in plant disease diagnoses and pathogen identification $[13,14]$. Lawler (1993) [15] used a serological technique to distinguish B. xylophilus from B. mucronatus. The results suggested that antiserum to B. xylophilus could distinguish the two species on Western blots, but that polyclonal antibodies did not distinguish the two species clearly using an ELISA system. This was due to the poor specificity of the polyclonal antibody. Thus, this method has not become popular.

To date, various molecular techniques have been developed for differentiating B. xylophilus from B. mucronatus, using polymerase chain reaction-restriction fragment length polymorphism (PCR-RFLP), random amplified polymorphism DNA (RAPD) techniques, species-specific primers based on internal transcribed spacers (ITS), and SCAR molecular marker, satDNA and Hsp70 genes [16-23]. Recently, a methodology using loop-mediated isothermal amplification (LAMP) was developed for the direct detection of PWN [24]. However, these detection techniques mentioned are based on nucleic acids.

The major goal of comparative proteomics is to determine proteomic differences in the same species in different developmental stages or between allied species. As with many ecologically important species, proteomics research in B. xylophilus lags far behind that in other nematodes, such as Caenorhabditis elegans (Maupas) Dougherty and Brugia malayi Brug [25,26]. The surface coat proteins of the PWN expressed during host pine infection and in vitro culture have been compared using a proteomics approach [27]. The secretome of B. xylophilus was analysed by a proteomics method combined with the available genomic sequence. The study revealed the tangled roots of 
parasitism and the potential for molecular mimicry [28]. However, little research on the differential proteomics of PWN and the related species B. mucronatus has been reported. Moreover, few scholars have attempted to identify PWN-specific proteins to develop a detection method for B. xylophilus. Most genes in the genome achieve their function through protein expression, modification and interaction. Thus, the analysis of the proteins is important for investigation of the molecular mechanisms of the phenomena of life [29].

In the present study, to gain a better understanding of proteins differentially expressed between B. xylophilus and B. mucronatus, a proteomics approach was used to identify a specific protein of PWN. 2-DE coupled with MALDI-TOF/TOF-MS was used to separate and identify proteins from the nematodes. Protein spots that were specifically expressed by B. xylophilus with relatively high abundance were selected for a series of studies. The specificity of the differentially expressed proteins was confimed using PCR with the genomic DNA from other nematode species. Subsequently, in situ hybridisation was used to identify sites of expression. The gene encoding the specific protein identified was cloned and expressed. RNAi was used to evaluate the function of the Bx-Prx gene. The specificity of the $B$. xylophilus protein identified and the encoding gene will facilitate development of detection technologies for B. xylophilus.

\section{Results and Discussion}

\subsection{Comparative Proteome Analysis and Protein Identification}

To identify PWN-specific proteins, we performed a comparative proteomics analysis. In this study, the focus was on B. xylophilus and B. mucronatus, which are closely related; however, the latter is considered to have very low virulence. Representative 2-DE gels of protein spots from the nematodes are shown in Figure 1. Approximately 2000 protein spots were detected on the 2-DE gel (Figure 1). The pattern revealed a broad distribution of spots in the pI range of 5 to 8 with an apparent molecular mass of 14.4-94.0 kDa. Examination of the 2-DE gels using the Image Master 2D Platinum 6.0 software (GE Healthcare, Uppsala, Sweden) revealed 35 protein spots with significant intensity differences, $>1.75$, between the two nematodes. Of them, 15 protein spots specifically expressed in B. xylophilus with relatively high abundance were excised from the gels and analyzed by MALDI-TOF/TOF. Database search results are listed in Table 1. The proteins identified, including actin, chaperonin Cpn60, GAPDH-1, aldolase, heat shock protein 70, aalectin-1, cytosolic fatty-acid binding, elongation factor 2, aldo/keto reductase, and peroxiredoxin, are involved in several processes, including cytoskeleton organization, protein folding, glycolysis, stress response, fruiting body development, transcription, ethanol oxidation and defense response.

The ability of proteomics to identify proteins is limited by the availability of sequence data [30]; thus the published genome data of PNW [31] are an important resource in the identification of proteins from this unusual parasite. 
Figure 1. Two-dimensional sodium dodecyl sulfate-polyacrylamide gel electrophoresis (SDS-PAGE) gels of B. xylophilus and B. mucronatus (A) B. xylophilus; and (B) B. mucronatus Proteins $(120 \mu \mathrm{g})$ of B. xylophilus and B. mucronatus extracts were separated, in the first dimension by isoelectric focusing $(\mathrm{pH} \mathrm{5-8)}$ and in the second dimension by SDS-PAGE in $12.5 \%$ acrylamide gels. Proteins were visualised by silver staining. Proteins indicated by arrows were specifically expressed by B. xylophilus and identified by MALDI-TOF/TOF.

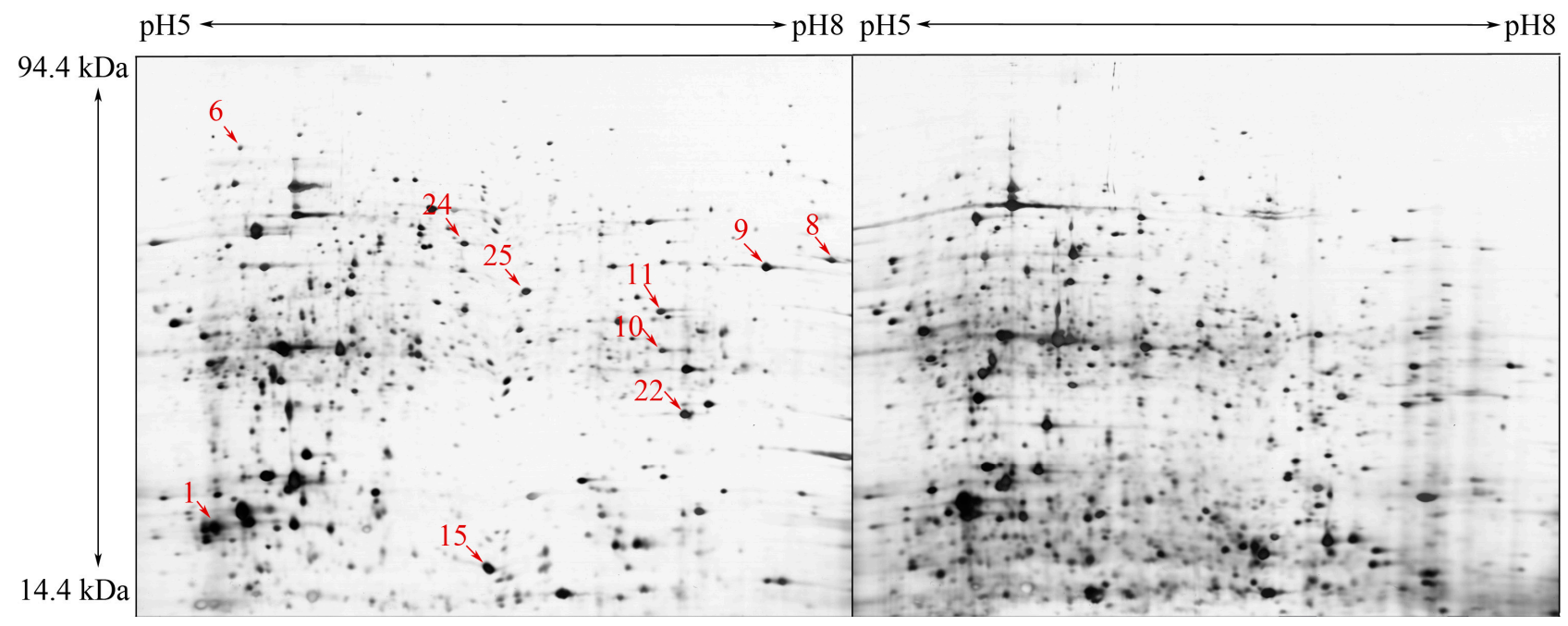

Table 1. Identification of specifically expressed proteins induced in B. xylophilus.

\begin{tabular}{|c|c|c|c|c|c|c|}
\hline $\begin{array}{l}\text { Spot } \\
\text { No. }\end{array}$ & Accession No. ${ }^{a}$ & Protein Name & $\mathbf{p I} / \mathbf{k D a}^{\mathrm{b}}$ & Score $^{c}$ & $\mathbf{P N}^{\mathbf{d}}$ & Molecular Function \\
\hline 1 & BUX.s00713.141 & Actin & $42 / 5.3$ & 264 & 6 & $\begin{array}{l}\text { ATP binding, structural } \\
\text { constituent of cytoskeleton }\end{array}$ \\
\hline 6 & BUX.s00789.28 & Chaperonin Cpn60 & $60 / 5.39$ & 213 & 13 & ATP binding \\
\hline 8 & BUX_s01281.46 & GAPDH-1 & $37 / 7.68$ & 419 & 10 & NAD+ activity \\
\hline 9 & BUX.s01438.76 & Aldolase & $40 / 7.66$ & 120 & 2 & $\begin{array}{l}\text { fructose-bisphosphate } \\
\text { aldolase activity }\end{array}$ \\
\hline 10 & BUX.s01653.149 & Heat shock protein 70 & $71 / 5.55$ & 528 & 14 & ATP binding \\
\hline 11 & BUX.s01109.344 & Galectin & $33 / 6.22$ & 146 & 5 & galactoside binding \\
\hline 15 & BUX_s01226.18 & $\begin{array}{l}\text { Cytosolic } \\
\text { Fatty-acid binding }\end{array}$ & $88 / 6.14$ & 71 & 9 & transporter activity \\
\hline 24 & BUX.s00397.100 & Elongation factor 2 & $96 / 6.4$ & 280 & 14 & $\begin{array}{l}\text { translation elongation } \\
\text { factor activity }\end{array}$ \\
\hline 25 & BUX.s01143.143 & Aldo/keto reductase & $37 / 6.22$ & 40 & 3 & oxidoreductase activity \\
\hline 22 & BUX.s01109.415 & Peroxiredoxin & $22.1 / 6.09$ & 156 & 6 & $\begin{array}{l}\text { thioredoxin peroxidase } \\
\text { activity }\end{array}$ \\
\hline
\end{tabular}

${ }^{a}$ Database accession numbers according to BUX.v1.2.genedb.protein.fa; ${ }^{\mathrm{b}} \mathrm{pI} / \mathrm{kDa}$ values were retrieved from the protein database; ${ }^{c}$ Mascot score reported after searching against the geneDB database at: www.genedb.org/ Homepage/Bxylophilus and ${ }^{\mathrm{d}}$ Number of peptides sequenced. 


\subsection{PCR Detection for Specificity}

According to mass spectrometry results and the B. xylophilus genome data, five genes encoding actin (spot 1), aldolase (spot 9), galectin-1 (spot 11), peroxiredoxin (spot 22), and elongation factor 2 (spot 24) were successfully amplified from B. xylophilus genomic DNA by PCR (Figure 2). Subsequently, the specificity of the five genes was assessed by PCR using genomic DNA from other nematodes. The results suggested that only the gene encoding peroxiredoxin (Bx-Prx) was specific to B. xylophilus (Figure 3). Various strains of B. xylophilus yielded amplification products of $749 \mathrm{bp}$, while strains of $B$. mucronatus and other nematodes showed no amplification. Therefore, the Bx-Prx gene was unique at the nucleotide sequence level.

Figure 2. Amplified fragments of genomic DNAs of B. xylophilus and B. mucronatus using specific primers. (A): aldolase; $(\mathbf{B})$ : actin-4; (C): elongation factor 2; (D): galectin-1 and (E): thiol peroxiredoxin (M): DL2000 marker; 1, 2, 3, genomic DNA of B. xylophilus, B. mucronatus and $\mathrm{ddH}_{2} \mathrm{O}$ as templates, respectively).

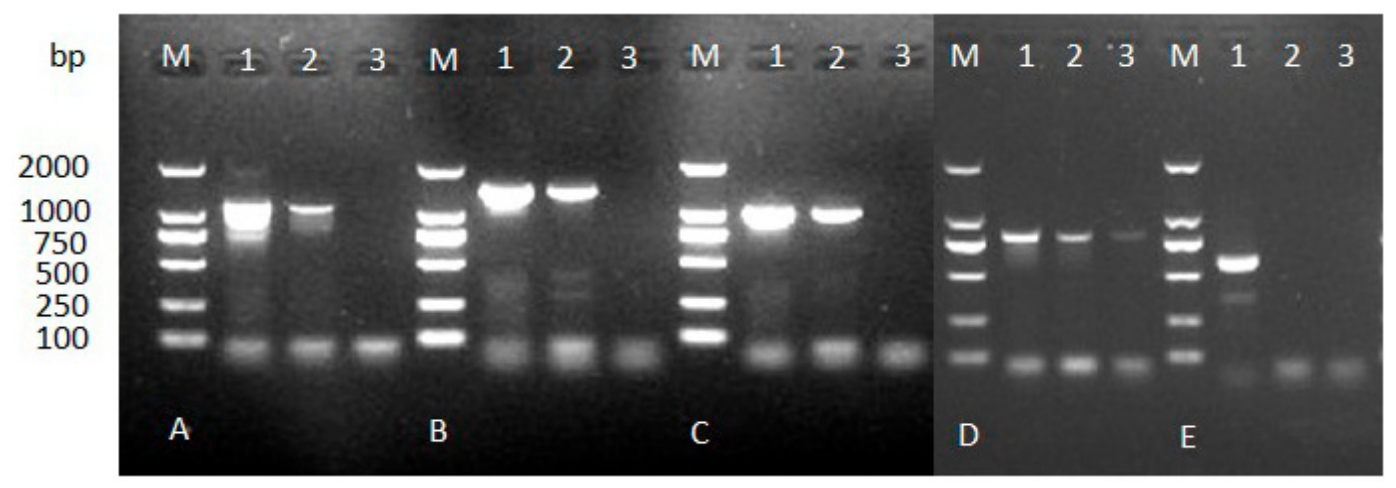

Figure 3. PCR amplification of genomic DNA of B. xylophilus, B. mucronatus and other nematode species using $B x$-Prx gene-specific primers (Bx-PrxF1/R1). Only B. xylophilus had an amplification product at 749 bp. Lanes 1-2, B. xylophilus strains; Lanes 3-5, B. mucronatus strains; Lanes 6-8, B. hofmanni (SF2), Seinura wuae (JY13), Aphelenchoides macronucleatus (GHHC2).

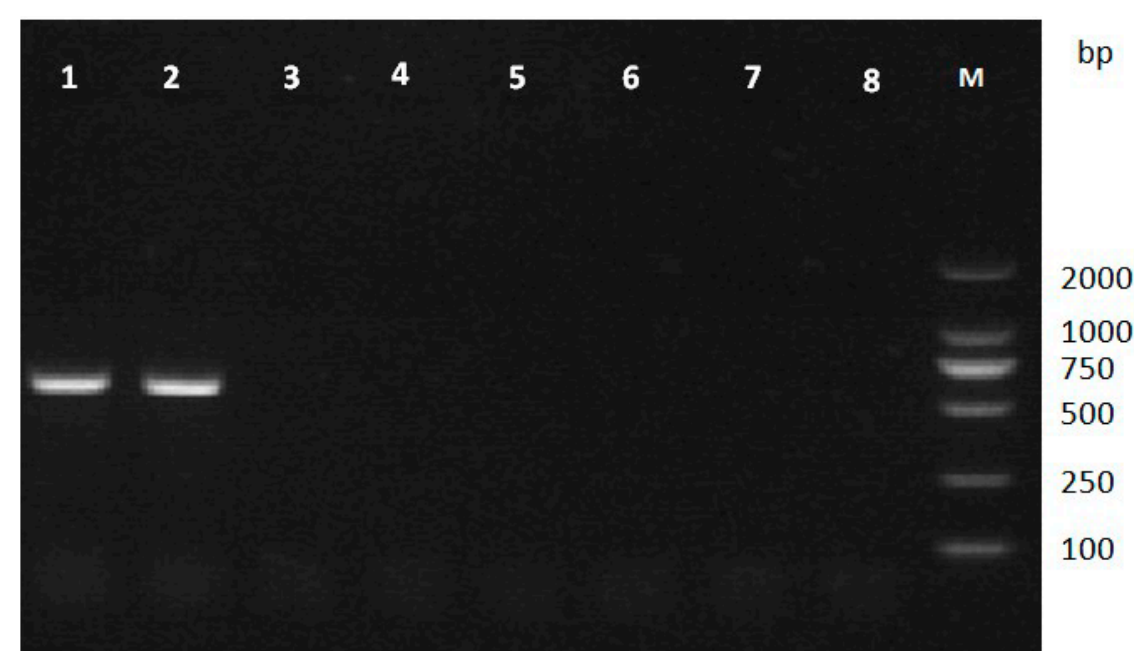


This might have been because the expression levels of the other four genes in B. mucronatus were below the detection limit of 2-DE. RT-PCR and quantitative real-time RT-PCR enabled identification of two genes expressed in a stage-specific manner (AC-cathB-1, AC-cathB-2) in Angiostrongylus cantonensis (Chen). $A C$-cath $B-1$ and $A C$-cathB-2 were expressed in L1 and L3, respectively, suggesting that $A C$-cath $B-1$ and $A C$-cath $B-2$ may play an important role in intermediate and final host invasion [32].

\subsection{Expression, Purification and Identification of Bx-Prx}

To further analyse the Bx-Prx protein, the encoding gene was cloned and expressed in a prokaryotic expression system. Total protein samples from IPTG-induced E. coli cells were sonicated and separated by SDS-PAGE. The gel revealed a $26-\mathrm{kDa}$ protein in the supernatant and pellet of bacteria harbouring $B x-P r x /$ pET-28a (+), consistent with the expected molecular weight of Bx-Prx. This result suggested that the recombinant protein exits $E$. coli in a soluble form and in inclusion bodies. No new band was evident in the control group (Figure 4). After nickel-nitrilotriacetic acid (NiNTA) purification, the purified recombinant protein was assessed by Western blotting using its $6 \times$ His sequence (Figure 5). A polyclonal antiserum against Bx-Prx recognized the target Bx-Prx as a single band, in only the extract from B. xylophilus not B. mucronatus [33]. Therefore, the purified Bx-Prx protein can be used as the candidate diagnostic antigen of pine wilt disease, facilitating development of immunological PWN detection methods. It was reported that two Prx2 genes from the parasitic nematode, Haemonchus contortus (Rudolphi) Cobb was recombinantly expressed, whose result indicated that the H. contortus Prx2 gene played an important role in hydrogen peroxide instability [34].

Figure 4. SDS-PAGE of the expressed product for pET 28a (+)-Bx-Prx in E. coli BL21(DE3). Lane 1: molecular weight markers (40, 25, 15, $10 \mathrm{kDa})$; Lane 2: soluble fraction of induced cells transformed by pET-28a; Lane 3: soluble fraction of induced cells transformed by pET $28 \mathrm{a}(+)$; Lane 4: inclusion body fraction of induced cells transformed by pET-28a and Lane 5: inclusion bodies fraction of induced cells transformed by pET-28a (+)-Bx-Prx; Lane 6: the purified pET 28a (+)-Bx-Prx recombinant protein.

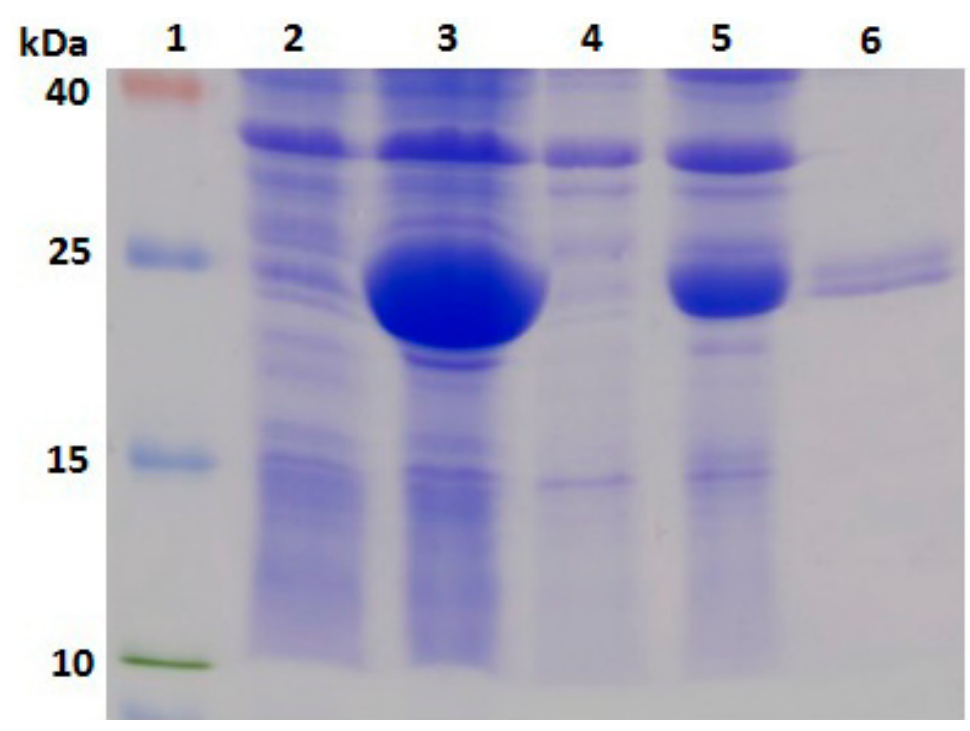


Figure 5. Western blots of protein purified from E. coli BL21(DE3) harbouring pET 28a (+)-Bx-Prx. Lane 1: molecular weight markers $(25,15,10,4.6 \mathrm{kDa})$ and Lane 2: Western blots of purified proteins.

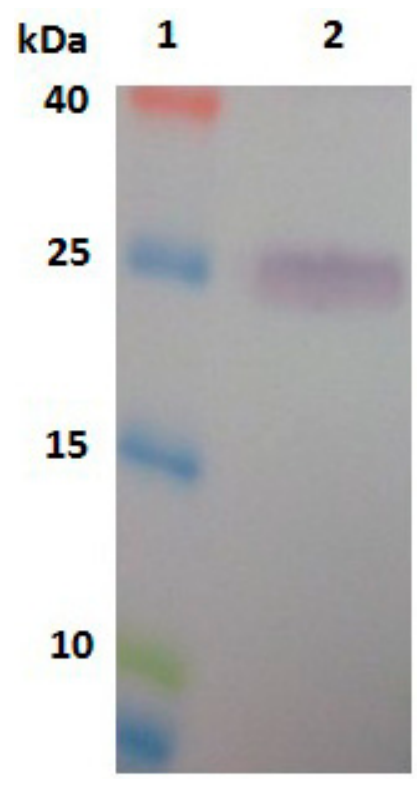

\subsection{In Situ Hybridisation}

In situ hybridisation (ISH) enables investigation of gene function in nematodes [35-37]. In this study, ISH was used to analyse expression patterns of the gene encoding the Bx-Prx protein. A digoxigenin-labeled probe generated from $B x-\operatorname{Prx}$ specifically hybridised in the tail of B. xylophilus (Figure 6a,b). No hybridisation was observed in B. mucronatus (Figure 6d) or the control group (Figure 6c). Therefore, Bx-Prx was specific to B. xylophilus and apparently absent from B. mucronatus. The immunohistolocalisation study using a polyclonal antiserum against recombinant $B x$-Prx suggested that the protein was localized under the cuticle of the nematode and in muscle cells and particles [33]. Thus, Bx-Prx is expressed under the cuticle of the nematode tail.

Superoxide dismutase, catalase, glutathione peroxidase and peroxiredoxin are major antioxidants in many organisms. The peroxiredoxins (Prxs) are ubiquitous thiol-specific peroxidases with catalytic functions in the detoxification of cytotoxic peroxides. The Prxs constitute a family of haem-free peroxidases with multiple functions. Their major functions can be divided into the following three categories: antioxidant activity by reducing alkyl peroxide and hydrogen peroxide to alcohol or water [38,39]; protection against phospholipid peroxidation [40] and protection of cells from oxidant-induced membrane damage and prevention of cell death [41]. The crucial role of peroxiredoxins in peroxide metabolism has been established in bacteria, plants and parasitic nematodes $[34,42,43]$. Most of these studies showed that the Prxs exhibited peroxidase activity or switched to a molecular chaperone upon heat shock or oxidative stress. Recently, relevant research on peroxiredoxins in the pine wood nematode demonstrated that the Bx-Prx protein plays an important role in protecting PWN against host immune responses [33]. Bx-Prx is one of 12 antioxidant proteins identified in the secretome of $B$. xylophilus. Futhermore, Bx-Prx was suggested to be basically a somatic protein and was also leaked from the PWN body. Given this background, we suggest that the 
secreted antioxidant Bx-Prx might play a role in protecting B. xylophilus from oxygen free radicals in the pine tree [28]. While B. xylophilus and B. mucronatus are closely related species, B. mucronatus has low virulence or no pathogenicity to host pine trees. Thus, the pathogenic differences between the species might be related to the differential expression of pathogenicity-associated genes. However, while our data suggests that Bx-Prx was expressed differentially between the two parasites; further studies are needed to determine the role of the $B x$-Prx gene in parasite-host interactions.

Figure 6. Localisation of Bx-Prx in the sexual gland. (A) B. xylophilus, hybridisation site was showed in the red table; (B) details of hybridisation signal of B. xylophilus; (C) B. xylophilus hybridised without a labelled probe and (D) B. mucronatus. Scale bars $=50 \mu \mathrm{m}$ in $(\mathbf{A}, \mathbf{C}, \mathbf{D})$ and $20 \mu \mathrm{m}$ in $(\mathbf{B})$.

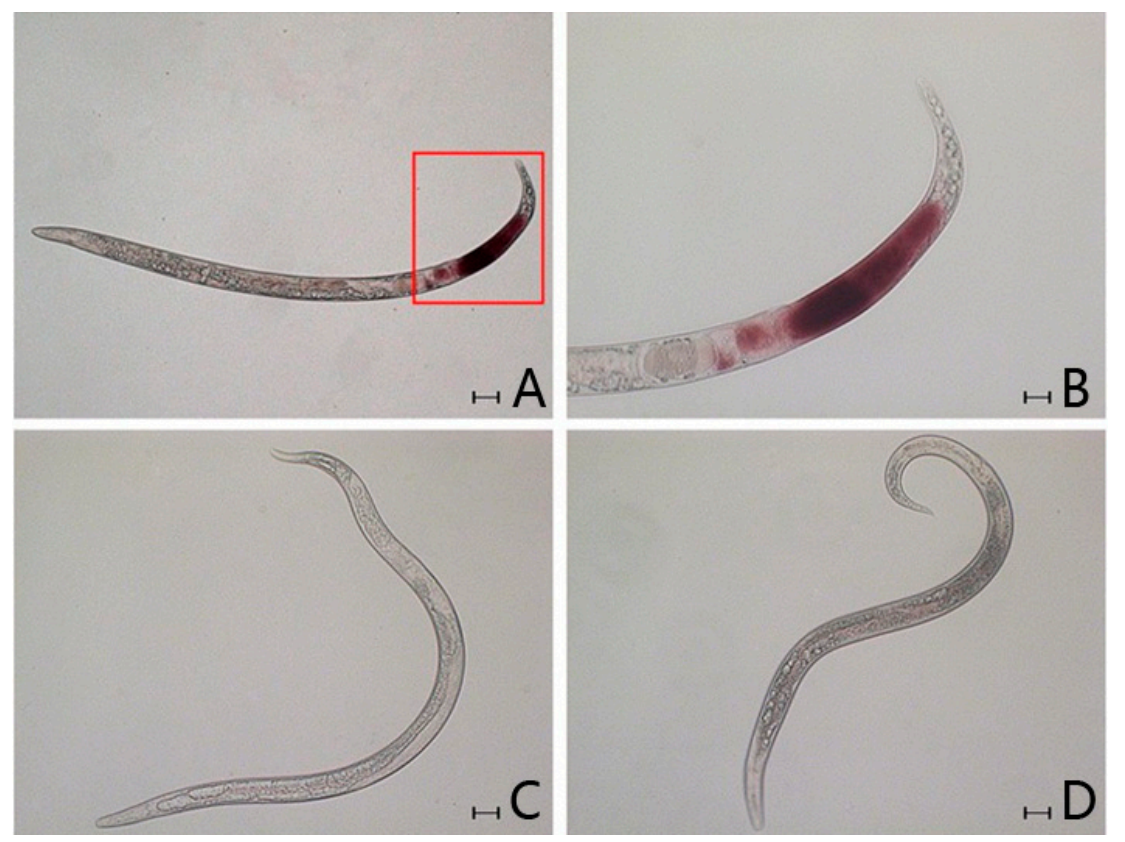

\subsection{Effect of RNAi on B. xylophilus Reproduction and Pathogenicity}

RNA interference (RNAi) was first described by Fire et al. (1998) [44] in C. elegans for analysis of gene function in vitro. In this study, RNAi was used to assess the function of Bx-Prx in B. xylophilus.

The effect of RNAi on B. xylophilus propagation was tested on PDA plates inoculated with B. cinerea. The nematode soaked in double-strain RNA (dsRNA) solution showed significantly reduced propagation compared with the control. The number of nematodes in the dsRNA treatment was 2867 compared to 6100 after 7 days (Figure 7). These results indicated that feeding and reproduction of $B$. xylophilus were influenced by the RNAi treatment.

q-PCR was performed to determine the effect of RNAi on the Bx-Prx mRNA level. The actin gene of $B$. xylophilus was used as a reference gene. Soaking of nematodes in dsRNA solution resulted in a marked decrease in Bx-Prx gene expression compared to the control (Figure 8). Taking the mRNA expression level of the control as $100 \%$, the mean expression level of dsRNA-treated samples was $0.071 \%(p<0.001)$. These result suggest that knockdown of the Bx-Prx gene by RAi is effective. 
Figure 7. Propagation of B. xylophilus washed from the PDA plate of $B$. cinerea with and without dsRNA treatment. $B x-\operatorname{Prx}$ and $\mathrm{CK}$ indicate the numbers of nematodes with and without dsRNA treatment, respectively. Bars show standard errors of the mean. The different letters on top of the bars $(a, b)$ indicate statistically significant differences was found between the dsRNA-treated and controls $(p<0.05)$.

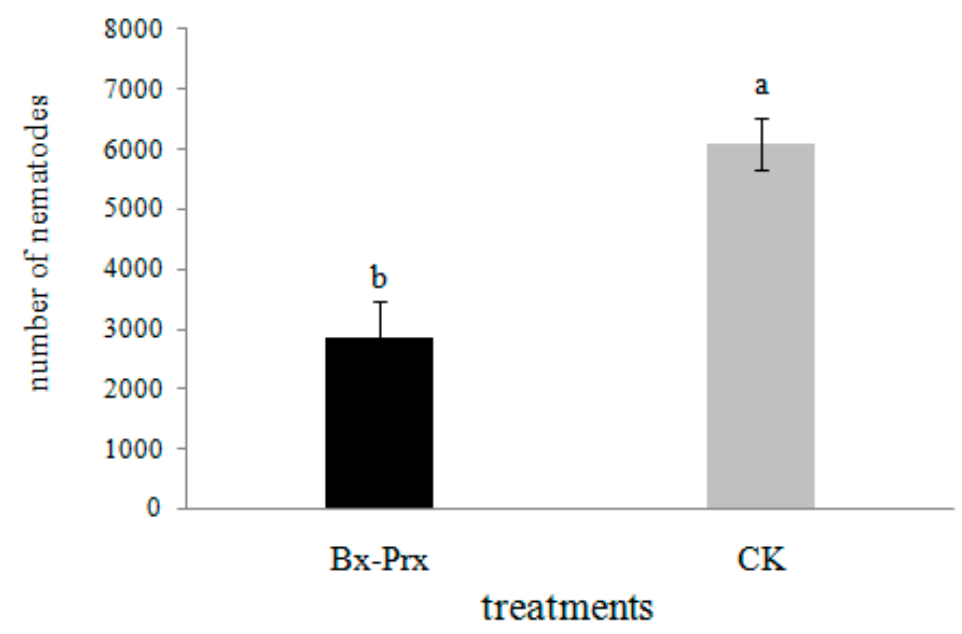

Figure 8. Bx-Prx mRNA levels in B. xylophilus after soaking in target dsRNA solution relative to the control (double-distilled water (DDW) with no dsRNA soaked). The expression level of the control was taken as $100 \%$. Bars show standard errors of the mean. The different letters on top of the bars $(a, b)$ indicate statistically significant differences was found between the dsRNA-treated and controls $(p<0.05)$.

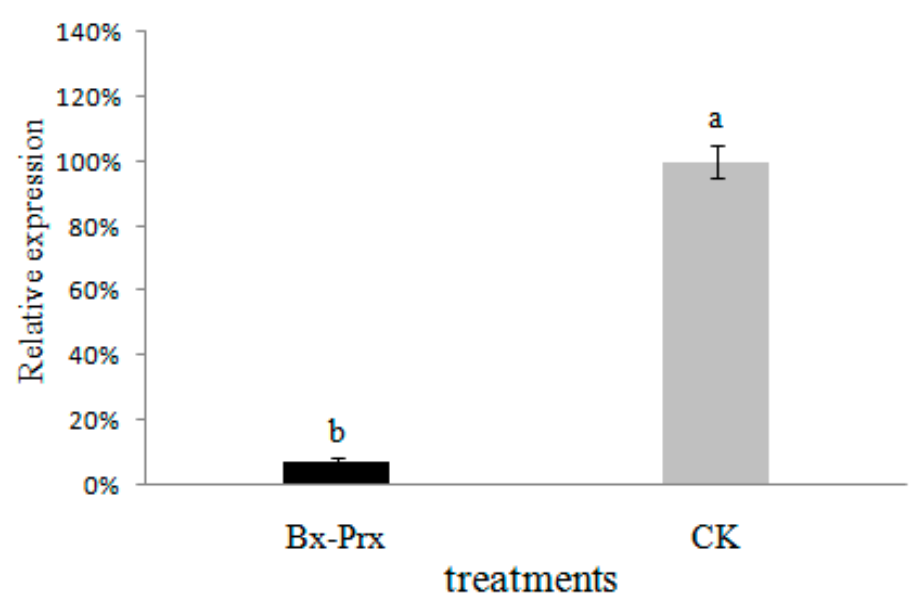

In 2-year-old $P$. thunbergii seedlings, inoculation with dsRNA-treated nematodes resulted in wilting and red-brown cones (Figure 9A). In contrast, only part of the plants inoculated with control nematodes wilted with some cones losing their green color (Figure 9B). These results indicated that B. xylophilus with down-regulated peroxiredoxin could result in a loss of pathogenicity.

Recently, RNAi has been applied to the investigation of arthropods [45], insects [46] and plant parasitic nematodes [47]. Moreover, RNAi has also been used to assess the pathogenic and molecular effects of the silenced B. xylophilus genes [48-50]. In this study, knockdown of the Bx-Prx gene markedly decreased the feeding, reproduction and pathogenicity of $B$. xylophilus. These results suggest that the Bx-Prx gene, knockdown of which results in the loss of pathogenicity, is important in PWN. 
It was reported that the RNAi efficiency by soaking may have worn off after a period of time [50-53], so further studies focus on the persistence of RNA interference are needed. However, the mechanisms by which it affects reproduction and pathogenicity will be addressed in future research.

Figure 9. Symptoms in $P$. thunbergii seedlings 0 (A) and 30 days (B) after inoculation with nematodes soaked in dsRNA solution (Line 3) and double-distilled water (DDW) (Line 2). P. thunbergii seedlings inoculated with DDW alone was used as controls (Line 1).

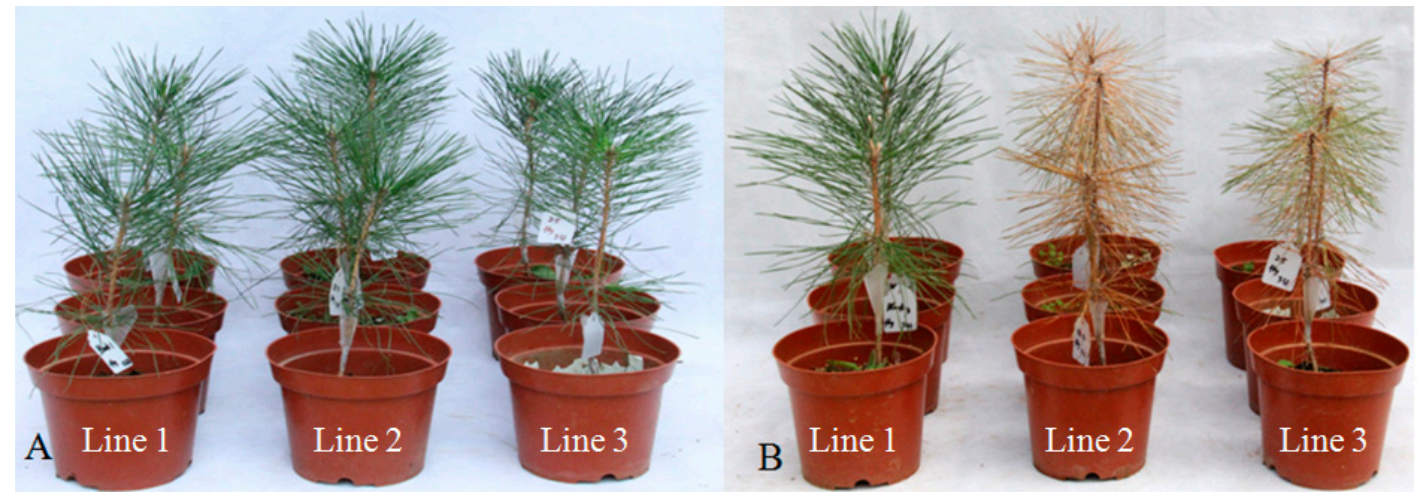

\section{Experimental Section}

\subsection{Experimental Organisms}

B. xylophilus and B. mucronatus were isolated from P. massoniana by the Key Laboratory of Pest Prevention and Control of Jiangsu Province, Nanjing, China. The nematodes were cultured for 1 week at $25{ }^{\circ} \mathrm{C}$ on Botrytis cinerea Pers. grown on autoclaved barley grains and were separated from B. cinerea hyphae on a Baermann funnel for $6-8 \mathrm{~h}$ at $25^{\circ} \mathrm{C}$ according to the method of Kikuchi et al. (2011). The nematodes were then washed five times in M9 buffer to remove any remaining $B$. cinerea mycelia, conidia, and hyphal fragments [31].

\subsection{Protein Extraction}

Proteins of a large number of nematodes were extracted according to the trichloroacetic acid (TCA)-acetone procedure described previously [54]. Briefly, nematodes were ground in a mortar with liquid nitrogen until a fine white powder was produced, and then suspended with $3 \mathrm{~mL} / \mathrm{g}$ fresh weight ice-cold acetone containing 10\% TCA and $0.07 \%$ dithiothreitol (DTT). The resulting protein-containing suspension was allowed to precipitate overnight at $-20{ }^{\circ} \mathrm{C}$ and then centrifuged $(20,000 \times \mathrm{g}, 20 \mathrm{~min})$. The pellet was rinsed three times with ice-cold acetone containing $0.07 \%$ DTT at $-20{ }^{\circ} \mathrm{C}$. Finally, the protein pellet was air-dried and dissolved in lysis buffer solution (8 M urea, 2\% CHAPS, 0.05\% immobilized $\mathrm{pH}$ gradient (IPG) buffer, $18 \mathrm{mM}$ DTT). The protein concentration was measured using 2-D Quant Kit (GE Healthcare, Uppsala, Sweden) and the solution was aliquotted into $1.5 \mathrm{~mL}$ tubes for storage at $-80{ }^{\circ} \mathrm{C}$. 


\subsection{IEF and SDS PAGE}

Each sample containing an estimated $120 \mu \mathrm{g}$ of protein in $450 \mu \mathrm{L}$ of IEF buffer was loaded onto a $24 \mathrm{~cm}$ immobilised pH 5-8 gradient strip (GE Healthcare Biosciences, Uppsala, Sweden). The strips were focused using a Ettan IPGphor Multiphor III (GE Healthcare, Uppsala, Sweden) at $20{ }^{\circ} \mathrm{C}$, applying the following program: $30 \mathrm{~V}$ for $6 \mathrm{~h}, 60 \mathrm{~V}$ for $6 \mathrm{~h}, 200 \mathrm{~V}$ for $1 \mathrm{~h}, 500 \mathrm{~V}$ for $1 \mathrm{~h}, 1000 \mathrm{~V}$ for $1 \mathrm{~h}$, $4000 \mathrm{~V}$ for $1 \mathrm{~h}, 8000 \mathrm{~V}$ gradient for $30 \mathrm{~min}$, and $8000 \mathrm{~V}$ for $64,000 \mathrm{Vh}$. After focusing, proteins were reduced in equilibration buffer (6 M urea, 29.3\% $w / v$ glycerol, $2 \% \mathrm{SDS}$, and $1.5 \mathrm{M}$ Tris- $\mathrm{HCl}, \mathrm{pH} 8.8$ ) containing $1 \% \mathrm{w} / \mathrm{v}$ DTT for $15 \mathrm{~min}$, followed by alkylation in a separate incubation for an additional $15 \mathrm{~min}$ in equilibration buffer containing $2.5 \% \mathrm{w} / \mathrm{v}$ iodoacetamide (IAA) instead of DTT. The strips were then transferred to $12.5 \%$ SDS PAGE gels for 2-DE using Amersham's Ettan DALT six gel system (GE Healthcare, Uppsala, Sweden) with SDS electrophoresis buffer (250 mM Tris, pH 8.3, $1.92 \mathrm{M}$ glycine, and $1 \% \mathrm{w} / v \mathrm{SDS}$ ) and $2 \mathrm{~W} /$ gel for $30 \mathrm{~min}$ and $17 \mathrm{~W} / \mathrm{gel}$ for $5 \mathrm{~h}$. At least three gels were run for each sample.

\subsection{Image Analysis}

Protein spots were detected by silver staining [55]. Stained gels were scanned and calibrated using the Labscan 5 software (GE Healthcare, Uppsala, Sweden). Three well-separated gels of each sample were used to create "replicate groups". Detection and matching of the protein spots was facilitated with the use of the Image Master 2D Platinum 6.0 (GE Healthcare, Uppsala, Sweden) software and re-evaluated by visual inspection. Spots that were expressed specifically by B. xylophilus in relatively high abundance were analysed by matrix-assisted laser desorption/ionisation-tandem time-of-flight mass spectrometry (MALDI-TOF/TOF). The UniProt database (http://www.uniprot.org) was searched to determine the functions of the proteins identified. The peptide mass data were analysed for corresponding protein matching in the geneDB database. A positive identification had to meet the following criteria: a significant MASCOT score and at least four matched peptides in MS analysis or two matched peptides in MALDI-TOF/TOF analysis.

\subsection{PCR Detection for Specificity of Differential Proteins}

According to the results of mass spectrometry and PWN genome-wide data, five genes (primers used for gene cloning are listed in Table 2) were amplified from B. xylophilus genomic DNA by polymerase chain reaction (PCR). Briefly, specific PCR reactions were conducted in $20 \mu \mathrm{L}$ volume, containing $2 \mu \mathrm{L}$ primers mixture $(10 \mu \mathrm{M}), 1 \mu \mathrm{L}$ DNA template, $10 \mu \mathrm{L}$ ExTaq mixture and $7 \mu \mathrm{L}$ sterile water. PCR reactions were performed with the following cycle conditions: (a) initial activation at $94{ }^{\circ} \mathrm{C}$ for $2 \mathrm{~min}$; (b) 35 cycles of $94{ }^{\circ} \mathrm{C}$ for $2 \mathrm{~min}, 55{ }^{\circ} \mathrm{C}$ for $45 \mathrm{~s}$, and $72{ }^{\circ} \mathrm{C}$ extension for $45 \mathrm{~s}$; and (c) a final extension at $72{ }^{\circ} \mathrm{C}$ for $10 \mathrm{~min}$. The amplified PCR products were confirmed by electrophoresis on $2 \%$ agarose gels. Gels were stained with ethidium bromide and viewed under a UV light. Then, the specificity of the five cloned genes was confirmed by PCR using genomic DNA from the other nematode species as the template. 
Table 2. Identification of specificallly expressed proteins induced in B. xylophilus.

\begin{tabular}{|c|c|c|c|c|}
\hline Gene & Spot No. & Sequence ID & Sense Primer (5-3) & Antisense Primer (5-3) \\
\hline \multirow{2}{*}{ Actin-4 } & \multirow{2}{*}{1} & \multirow{2}{*}{$\mathrm{gb} \mid \mathrm{ACZ13341.1|}$} & ATGTGTGACGAAGAAG & TTAGAAACATTTGCGGT \\
\hline & & & TTGCCGCTC & GAACGATG \\
\hline \multirow{2}{*}{ Aldolase } & \multirow{2}{*}{9} & \multirow{2}{*}{$\mathrm{gb} \mid \mathrm{ACZ13345.1|}$} & ATGGCCGAAGTCGGTG & TTAGTATGCGTGGTCTA \\
\hline & & & СТTCTTATC & CGAGAGGG \\
\hline \multirow{2}{*}{ Galectin-1 } & \multirow{2}{*}{11} & \multirow{2}{*}{ gb|ACZ13331.1| } & ATGACTGAGGAAAAGA & TTAATGGATCTGGATGC \\
\hline & & & AAACTTACA & CAGTGATC \\
\hline \multirow{2}{*}{$\begin{array}{c}\text { Elongation } \\
\text { factor } 2\end{array}$} & \multirow{2}{*}{24} & \multirow{2}{*}{$\mathrm{gb} \mid \mathrm{ACZ13348.1|}$} & CCGGAATTCATGAATC & CCCAAGCTTTTAGAGTT \\
\hline & & & CTTCGGTTTCACCGCTG & TATCGTAGAAGTTGTCG \\
\hline \multirow{2}{*}{$B x-\operatorname{Prx}$} & \multirow{2}{*}{22} & \multirow{2}{*}{$\mathrm{gb} \mid \mathrm{ABW} 81468.1$} & ATGTCCAAGGCTTTCA & TTAATGTTTGTTGAAAT \\
\hline & & & TTGGCAAAC & ATTCGTGA \\
\hline
\end{tabular}

\subsection{Expression, Purification and Identification of Bx-Prx}

The entire open reading frame of Bx-Prx was cloned using B. xylophilus cDNA as the template and specific primers (5'-CGGGATCCATGTCCAAGGCTTTCATTGGCA-3' containing a BamHI site, and 5'-CGAGCTCTTAATGTTTGTTGAAATATTCGT-3' containing a SacI site). The amplified products were digested with BamHI and SacI (TaKaRa, Dalian, China) and ligated to the similarly digested pET-28a (+) vector using T4 DNA ligase at $37^{\circ} \mathrm{C}$. E. coli BL21 (DE3) cells were transformed by heat shock with the ligation product and plated on a selective medium. Subsequently, a single colony of the transformants was inoculated and cultured at $37^{\circ} \mathrm{C}$ in LB medium containing kanamycin $(50 \mu \mathrm{g} / \mathrm{mL})$ with shaking $(200 \mathrm{rpm})$ until the optical density $\left(\mathrm{OD}_{600}\right)$ reached 0.6. Then, protein expression was induced by addition of isopropyl- $\beta$-D-thiogalactoside (IPTG) to a final concentration of $1 \mathrm{mM}$ at $18{ }^{\circ} \mathrm{C}$. Cultivation was continued for $20 \mathrm{~h}$. Then, $200 \mathrm{~mL}$ bacterial culture was pelleted by centrifugation $\left(8000 \times \mathrm{g}, 10 \mathrm{~min}, 4^{\circ} \mathrm{C}\right)$. Subsequently, the pellets were dissolved in Tris-HCl $(20 \mathrm{mM}, \mathrm{pH} 8.0)$ and sonicated on ice. The supernatant and pellet were collected after centrifugation for a second time. The supernatant was purified based on its His-tag by affinity chromatography using HisTrap FF crude (GE Healthcare, Uppsala, Sweden). The soluble fraction, the insoluble fraction, the purified recombinant protein and the control (bacterium containing pET-28a $(+)$ ) were analysed on a $12.5 \%$ SDS-PAGE gel. Western blot analysis was conducted to confirm the recombinant Bx-Prx protein using an antibody raised against the poly-His tag (anti-His mouse IgG, Tiangen, Beijing, China).

\subsection{In Situ Hybridisation}

In situ hybridisation was performed on the B. xylophilus and B. mucronatus essentially as described previously [56-58]. Hybridisation probes were generated by amplification of the full length cDNA of Bx-Prx. PCR products were purified with the PCR Product Clean up Kit (Axygen Scientific, Inc., Union, CA, USA) and then random primer-labelled with digoxigenin using the DIG High Prime DNA Labeling and Detection Starter Kit I (Roche Applied Science, Mannheim, Germany). Then, the labelled probe was purified with the QIAquick Nucleotide Removal Kit (QIAGEN, Valencia, CA). In situ hybridisation was performed with mixed-stage B. xylophilus and B. mucronatus on poly-lysine-treated microslides. Nematodes were pre-treated with proteinase $\mathrm{K}(10 \mu \mathrm{g} / \mathrm{mL})$ for $30 \mathrm{~min}$ at $37{ }^{\circ} \mathrm{C}$ before the post-hybridization washing step. Hybridisation and detection were performed with the DIG-High Prime 
DNA Labelling and Detection Starter Kit I (RocheDiagnostics, Mannheim, Germany), and examined using a Zeiss Axio Image M2 microscope (Zeiss MicroImaging GmbH, Oberkochen, Germany).

\subsection{Bx-Prx Knockdown by RNA Interference (RNAi)}

The nematodes were ground in a mortar with liquid nitrogen until a fine white powder was produced. Total RNA was extracted from the resulting powder with Trizol reagent (Invitrogen, CA, USA). The RNA was then reverse-transcribed using a cDNA synthesis Kit (TransGen Biotech, Beijing, China), according to the manufacturer's protocol. Double-stranded RNA (dsRNA) was synthesised using the MEGscript RNAi Kit (Ambion Inc., Austin, TX, USA) with the primer set (Bx-Prx) designed in Section 2.5. The RNAi soaking method was performed basically according to Urwin et al. (2002) [59]. Freshly cultured nematodes of $B$. xylophilus (a mix of adults and juveniles, approximately 3000 individuals) were soaked in $30 \mu \mathrm{L}$ dsRNA solution and incubated at $180 \mathrm{rpm}$ for $24 \mathrm{~h}$ at $20^{\circ} \mathrm{C}$. B. xylophilus soaked in DDW without dsRNA were used as the negative control. After soaking, samples from each treatment were washed thoroughly several times in DDW and then used for further experiments. First, 15 pairs of female and male nematodes were picked and transferred onto a potato dextrose agar (PDA) plate with $B$. cinerea and cultured at $25{ }^{\circ} \mathrm{C}$ for 7 days. Then, the worms were washed from the plate and the nematodes were counted. Second, approximately 200 nematodes were used to verify the efficacy of Bx-Prx knockdown by qPCR carried out using a Kit (TransStart Green qPCR SuperMix; TransGen Biotech, Beijing, China) and a thermal cycler (ABI Prism 7500, Applied Biosystems, Foster City, CA, USA), with the following primers for Bx-Prx: 5'-GCTTTCCGTGGTTTG TTC-3' and 5'-ACTTCTCCGTGCTTGTCG-3' (from nucleotides 367-506). The cycling profile used was: $94{ }^{\circ} \mathrm{C}$ for $30 \mathrm{~s}$, followed by 40 cycles of $94{ }^{\circ} \mathrm{C}$ for $5 \mathrm{~s}$; and $60{ }^{\circ} \mathrm{C}$ for $34 \mathrm{~s}$. Actin was used as a reference gene. Initial data analysis was performed using the ABI Prism 7500, which calculated

$C_{\mathrm{t}}$ values and extrapolated relative levels of PCR products from standard curves. The $2^{-\Delta \Delta C \mathrm{t}}$ method was used to quantify the relative changes in gene expression. qPCR was conducted for three biological replicates, which included three technical replicates per reaction. Finally, the remaining nematodes were subjected to pathogenicity determination according to a previous method [60] with inoculation onto 2-year-old Pinus thunbergii seedlings. Thus, 1000 nematodes were injected into each seedling. B. xylophilus soaked in DDW without dsRNA and DDW alone, were used as controls. Periodically, we observed the seedlings and took photographs to record their state. Three biological replicates were conducted.

\section{Conclusions}

In conclusion, using proteomic technologies, we identified a specific B. xylophilus protein encoded by a gene, which is apparently present only in B. xylophilus. The identification of this gene, cloned and expressed in E. coli, provides fundamental information for identifying B. xylophilus via molecular approaches. In situ hybridisation pattern of $B x$-Prx showed that it was expressed in the tail of B. xylophilus. RNAi was used to evaluate the function of $B x-\operatorname{Pr} x$, the results indicated that the gene was associated with the reproduction and pathogenicity of B. xylophilus. This discovery provides fundamental information for identifying B. xylophilus via molecular approaches. Furthermore, the 
purified recombinant protein has potential for use as a candidate diagnostic antigen of pine wilt disease, which may lead to development of a new immunological detection method for PWN.

\section{Acknowledgments}

This work was financially supported by the Special Research Program for Forestry Sectors Beneficial to the Public of the China State Forestry Administration (201204501), Graduate Scientific Research and Innovation Plan of Universities in Jiangsu Province, China (CXZZ12-0520) and National Natural Science Foundation of China (31000303).

\section{Author Contributions}

Hanyu Fu: study design, guarantor of integrity of entire study, experimental studies, manuscript preparation; Jiahong Ren: manuscript revision, data analysis, literature research; Lin Huang: data acquisition, manuscript editing; Hao Li: data acquisition, manuscript editing; and Jianren Ye: guarantor of integrity of entire study, manuscript final version approval.

\section{Conflicts of Interest}

The authors declare no conflict of interest.

\section{References}

1. Jones, J.T.; Moens, M.; Mota, M.; Li, H.; Kikuchi, T. Bursaphelenchus xylophilus: Opportunities in comparative genomics and molecular host-parasite interactions. Mol. Plant Pathol. 2008, 9 , 357-368.

2. Mamiya, Y. Pathology of the pine wilt disease caused by Bursaphelenchus xylophilus. Annu. Rev. Phytopathol. 1983, 21, 201-220.

3. Mota, M.M.; Braasch, H.; Bravo, M.A.; Penas, A.C.; Burgermeister, W.; Metge, K.; Sousa, E. First report of Bursaphelenchus xylophilus in Portugal and in Europe. Nematology 1999, 1, 727-734.

4. Penas, A.C.; Correia, P.; Bravo, M.A.; Mota, M.; Tenreiro, R. Species of Bursaphelenchus Fuchs, 1937 (Nematoda: Parasitaphelenchidae) associated with maritime pine in Portugal. Nematology 2004, 6, 437-453.

5. Cao, A.; Liu, X.; Zhu, S.; Lu, B. Detection of the pinewood nematode, Bursaphelenchus xylophilus, using a real-time polymerase chain reaction assay. Phytopathology 2005, 95, 566-571.

6. Mamiya, Y.; Enda, N. Bursaphelenchus mucronatus n. sp. (Nematoda: Aphelenchoididae) from pine wood and its biology and pathogenicity to pine trees. Nematologica 1979, 25, 353-361.

7. Braasch, V.H. Pathogenitätstests mit Bursaphelenchus mucronatus an Kiefern und Fichtensämlingen in Deutschland. Eur. J. For. Pathol. 1996, 26, 205-216.

8. Tomminen, J. Pathogenicity studies with Bursaphelenchus mucronatus in Scots pine in Finland. Eur. J. For. Pathol. 1993, 23, 236-243.

9. Wingfield, M.; Blanchette, A.; Kondo, E. Comparison of the pine wood nematode, Bursaphelenchus xylophilus from pine and balsam fir1. Eur. J. For. Pathol. 1983, 13, 360-372. 
10. Bolla, R.; Wood, R. Pathogenicity and potential for spread of the pine wood nematode Bursaphelenchus spp. In Sustainability of Pine Forests in Relation to Pine Wilt and Decline; Proceedings of International Symposium: Tokyo, Japan, 1998; pp. 3-13.

11. Braasch, H.; Mota, M.; Vieira, P. Morphology of Bursaphelenchus xylophilus compared with other Bursaphelenchus species. In The Pinewood Nematode, Bursaphelenchus xylophilus, Proceedings of the International Workshop, Évora, Portugal, 20-22 August 2001; University of Évora: Évora, Portugal, August 2011.

12. Cardoso, J.M.; Fonseca, L.; Abrantes, I. Direct molecular detection of the pinewood nematode, Bursaphelenchus xylophilus, from pine wood, bark and insect vector. Eur. J. Plant Pathol. 2012, 133, 419-425.

13. Bartosch, S.; Hartwig, C.; Spieck, E.; Bock, E. Immunological detection of nitrospira-like bacteria in various soils. Microb. Ecol. 2002, 43, 26-33.

14. Lin, N.; Hsu, Y.; Hsu, H. Immunological detection of plant viruses and a mycoplasmalike organism by direct tissue blotting on nitrocellulose membranes. Phytopathology 1990, 80, 824-828.

15. Lawler, C.; Joyce, P.; Harmey, M. Immunological differentiation between Bursaphelenchus xylophilus and B. Mucronatus. Nematologica 1993, 1, 536-546.

16. Burgermeister, W.; Metge, K.; Braasch, H.; Buchbach, E. Its-RFLP patterns for differentiation of 26 Bursaphelenchus species (Nematoda: Parasitaphelenchidae) and observations on their distribution. Rus. J. Nematol. 2005, 13, 29.

17. Chen, F.M.; Negi, S.; Ye, J.R. A scar molecular marker to distinguish Bursaphelenchus mucronatus from the pinewood nematode, B. Xylophilus. For. Pathol. 2011, 41, 376-381.

18. Huang, L.; Ye, J.-R.; Wu, X.-Q.; Xu, X.-L.; Sheng, J.-M.; Zhou, Q.-X. Detection of the pine wood nematode using a real-time PCR assay to target the DNA topoisomerase I gene. Eur. J. Plant Pathol. 2010, 127, 89-98.

19. Irdani, T.; Marinari, A.; Bogani, P.; Ambrogioni, L.; Caroppo, S.; Buiatti, M. Molecular diversity among pine wood Bursaphelenchus populations detected by RAPD analysis. Redia 1995, 78, $149-161$.

20. Takemoto, S.; Kanzaki, N.; Futai, K. PCR-RFLP image analysis: A practical method for estimating isolate-specific allele frequency in a population consisting of two different strains of the pinewood nematode, Bursaphelenchus xylophilus (Aphelenchida: Aphelencoididae). Appl. Entomol. Zool. 2005, 40, 529-535.

21. Takeuchi, Y.; Kanzaki, N.; Futai, K. Nematode, Bursaphelenchus xylophilus, from pine wood. Nematology 2005, 7, 775-782.

22. Zheng, J.; Subbotin, S.A.; He, S.; Gu, J.; Moens, M. Molecular characterisation of some Asian isolates of Bursaphelenchus xylophilus and B. Mucronatus using PCR-RFLPs and sequences of ribosomal DNA. Rus. J. Nematol. 2003, 11, 17-22.

23. Zhuo, K.; Luo, M.; Cui, R.; Liao, J. A multiplex one-step PCR method for the simultaneous identification of Bursaphelenchus xylophilus, B. Mucronatus and B. Doui-three species within the xylophilus group. For. Pathol. 2011, 41, 66-69.

24. Kikuchi, T.; Aikawa, T.; Oeda, Y.; Karim, N.; Kanzaki, N. A rapid and precise diagnostic method for detecting the pinewood nematode Bursaphelenchus xylophilus by loop-mediated isothermal amplification. Phytopathology 2009, 99, 1365-1369. 
25. Bogaerts, A.; Temmerman, L.; Boerjan, B.; Husson, S.J.; Schoofs, L.; Verleyen, P. A differential proteomics study of Caenorhabditis elegans infected with Aeromonas hydrophila. Dev. Comp. Immunol. 2010, 34, 690-698.

26. Jiang, D.; Malone, J.; Townsend, R.; Weil, G.J.; Li, B. Multiplex proteomics analysis of gender-associated proteins in Brugia malayi. Int. J. Parasitol. 2012, 42, 841-850.

27. Shinya, R.; Morisaka, H.; Takeuchi, Y.; Ueda, M.; Futai, K. Comparison of the surface coat proteins of the pine wood nematode appeared during host pine infection and in vitro culture by a proteomic approach. Phytopathology 2010, 100, 1289-1297.

28. Shinya, R.; Morisaka, H.; Kikuchi, T.; Takeuchi, Y.; Ueda, M.; Futai, K. Secretome analysis of the pine wood nematode Bursaphelenchus xylophilus reveals the tangled roots of parasitism and its potential for molecular mimicry. PLoS One 2013, 8, e67377.

29. Seo, H.-S.; Hirano, M.; Shibato, J.; Rakwal, R.; Hwang, I.K.; Masuo, Y. Effects of coffee bean aroma on the rat brain stressed by sleep deprivation: A selected transcript-and 2D gel-based proteome analysis. J. Agric. Food Chem. 2008, 56, 4665-4673.

30. Fang, X.P.; Ma, H.S.; Lu, D.Z.; Yu, H.; Lai, W.; Ruan, S. Comparative proteomics analysis of proteins expressed in the I-1 and I-2 internodes of strawberry stolons. Prot. Sci. 2011, 9, 26-41.

31. Kikuchi, T.; Cotton, J.A.; Dalzell, J.J.; Hasegawa, K.; Kanzaki, N.; McVeigh, P.; Takanashi, T.; Tsai, I.J.; Assefa, S.A.; Cock, P.J. Genomic insights into the origin of parasitism in the emerging plant pathogen Bursaphelenchus xylophilus. PLoS Pathog. 2011, 7, e1002219.

32. Ni, F.; Wang, Y.; Zhang, J.; Yu, L.; Fang, W.; Luo, D. Cathepsin B-like and hemoglobin-type cysteine proteases: Stage-specific gene expression in Angiostrongy cantonensis. Exp. Parasitol. 2012, 131, 433-441.

33. Li, Z.; Liu, X.; Chu, Y.; Wang, Y.; Zhang, Q.; Zhou, X. Cloning and characterization of a 2-Cys peroxiredoxin in the pine wood nematode, Bursaphelenchus xylophilus, a putative genetic factor facilitating the infestation. Int. J. Biol. Sci. 2011, 7, 823.

34. Hudson, A.L.; Sotirchos, I.M.; Davey, M.W. The activity and hydrogen peroxide sensitivity of the peroxiredoxins from the parasitic nematode Haemonchus contortus. Mol. Biochem. Parasitol. 2011, 176, 17-24.

35. Balasubramanian, N.; Nascimento, G.; Ferreira, R.; Martinez, M.; Simões, N. Pepsin-like aspartic protease (Sc-ASP155) cloning, molecular characterization and gene expression analysis in developmental stages of nematode Steinernema carpocapsae. Gene 2012, 500, 164-171.

36. Lin, S.; Jian, H.; Zhao, H.; Yang, D.; Liu, Q. Cloning and characterization of a venom allergen-like protein gene cluster from the pinewood nematode Bursaphelenchus xylophilus. Exp. Parasitol. 2011, 127, 440-447.

37. Vandekerckhove, T.T.; Coomans, A.; Cornelis, K.; Baert, P.; Gillis, M. Use of the Verrucomicrobia-specific probe EUB338-III and fluorescent in situ hybridization for detection of "Candidatus xiphinematobacter" cells in nematode hosts. Appl. Environ. Microbiol. 2002, 68, 3121-3125.

38. Netto, L.E.; Chae, H.Z.; Kang, S.-W.; Rhee, S.G.; Stadtman, E.R. Removal of hydrogen peroxide by thiol-specific antioxidant enzyme (TSA) is involved with its antioxidant properties TSA possesses thiol peroxidase activity. J. Biol. Chem. 1996, 271, 15315-15321. 
39. Bruchhaus, I.; Richter, S.; Tannich, E. Removal of hydrogen peroxide by the $29 \mathrm{kDa}$ protein of Entamoeba histolytica. Biochem. J. 1997, 326, 785-789.

40. Manevich, Y.; Sweitzer, T.; Pak, J.H.; Feinstein, S.I.; Muzykantov, V.; Fisher, A.B. 1-Cys peroxiredoxin overexpression protects cells against phospholipid peroxidation-mediated membrane damage. Proc. Natl. Acad. Sci. 2002, 99, 11599-11604.

41. Kim, H.-S.; Pak, J.H.; Gonzales, L.W.; Feinstein, S.I.; Fisher, A.B. Regulation of 1-Cys peroxiredoxin expression in lung epithelial cells. Am. J. Respir. Cell Mol. Biol. 2002, 27, 227-233.

42. Kim, I.-S.; Kim, Y.-S.; Yoon, H.-S. Expression of salt-induced 2-Cys peroxiredoxin from Oryza sativa increases stress tolerance and fermentation capacity in genetically engineered yeast Saccharomyces cerevisiae. Appl. Microb. Biotechnol. 2013, 97, 3519-3533.

43. Turturice, B.A.; Lamm, M.A.; Tasch, J.J.; Zalewski, A.; Kooistra, R.; Schroeter, E.H.; Sharma, S.; Kawazu, S.-I.; Kanzok, S.M. Expression of cytosolic peroxiredoxins in Plasmodium berghei Ookinetes is regulated by environmental factors in the mosquito bloodmeal. PLoS Pathog. 2013, 9, e1003136.

44. Fire, A.; Xu, S.; Montgomery, M.K.; Kostas, S.A.; Driver, S.E.; Mello, C.C. Potent and specific genetic interference by double-stranded RNA in Caenorhabditis elegans. Nature 1998, 391, 806-811.

45. Wang, D.; Li, F.; Chi, Y.; Xiang, J. Potential relationship among three antioxidant enzymes in eliminating hydrogen peroxide in penaeid shrimp. Cell Stress Chaperones 2012, 17, 423-433.

46. Niu, B.L.; Shen, W.F.; Liu, Y.; Weng, H.B.; He, L.H.; Mu, J.J.; Wu, Z.L.; Jiang, P.; Tao, Y.Z.; Meng, Z.Q. Cloning and RNAi-mediated functional characterization of MaLac2 of the pine sawyer, Monochamus alternatus. Insect Mol. Biol. 2008, 17, 303-312.

47. Geldhof, P.; Murray, L.; Couthier, A.; Gilleard, J.S.; McLauchlan, G.; Knox, D.P.; Britton, C. Testing the efficacy of RNA interference in Haemonchus contortus. Int. J. Parasitol. 2006, 36, 801-810.

48. Ma, H.; Lu, Q.; Liang, J.; Zhang, X. Functional analysis of the cellulose gene of the pine wood nematode, Bursaphelenchus xylophilus, using RNA interference. Genet. Mol. Res.2011, 10, 1931-1941.

49. Kang, J.S.; Koh, Y.H.; Moon, Y.S.; Lee, S.H. Molecular properties of a venom allergen-like protein suggest a parasitic function in the pinewood nematode Bursaphelenchus xylophilus. Int. J. Parasitol. 2012, 42, 63-70.

50. Cheng, X.-Y.; Dai, S.-M.; Xiao, L.; Xie, B.-Y. Influence of cellulase gene knockdown by dsRNA interference on the development and reproduction of the pine wood nematode, Bursaphelenchus xylophilus. Nematology 2010, 12, 225-233.

51. Chhabra, A., Advances in genome engineering approaches. Adv. Genet. Eng. 2014, 3, e107.

52. Buehler, E.; Khan, A.A.; Marine, S.; Rajaram, M.; Bahl, A.; Burchard, J.; Ferrer, M. siRNA off-target effects in genome-wide screens identify signaling pathway members. Sci. Rep. 2012, 2 , doi:10.1038/srep00428.

53. Marine, S.; Bahl, A.; Ferrer, M.; Buehler, E. Common seed analysis to identify off-target effects in sirna screens. J. Biomol. Screen. 2012, 17, 370-378. 
54. Wang, X.; Chang, L.; Wang, G.; Sun, Z.; Ma, H.; Sun, Q.; Li, J. Protein extraction from the earthworm Eisenia fetida for 2-DE. Proteomics 2010, 10, 1095-1099.

55. Wang, X.; Chen, Y.; Han, Q.B.; Chan, C.Y.; Wang, H.; Liu, Z.; Cheng, C.H.K.; Yew, D.T.; Lin, M.; He, M.L. Proteomic identification of molecular targets of gambogic acid: Role of stathmin in hepatocellular carcinoma. Proteomics 2009, 9, 242-253.

56. De Boer, J.; Yan, Y.; Smant, G.; Davis, E.; Baum, T. In-situ hybridization to messenger RNA in Heterodera glycines. J. Nematol. 1998, 30, 309.

57. Wang, F.; Wang, Z.; Li, D.; Chen, Q. Identification and characterization of a Bursaphelenchus xylophilus (Aphelenchida: Aphelenchoididae) thermotolerance-related gene: Bx-hsp90. Int. J. Mol. Sci. 2012, 13, 8819-8833.

58. Kikuchi, T.; Shibuya, H.; Aikawa, T.; Jones, J.T. Cloning and characterization of pectate lyases expressed in the esophageal gland of the pine wood nematode Bursaphelenchus xylophilus. Mol. Plant-Microbe Interact. 2006, 19, 280-287.

59. Urwin, P.; Lilley, C.J.; Atkinson, H.J. Ingestion of double-stranded RNA by preparasitic juvenile cyst nematodes leads to RNA interference. Mol. Plant-Microbe Interact. 2002, 15, 747-752.

60. Zhu, L.-H.; Ye, J.; Negi, S.; Xu, X.-L.; Wang, Z.-L.; Ji, J.-Y. Pathogenicity of aseptic Bursaphelenchus xylophilus. PLoS One 2012, 7, e38095.

(C) 2014 by the authors; licensee MDPI, Basel, Switzerland. This article is an open access article distributed under the terms and conditions of the Creative Commons Attribution license (http://creativecommons.org/licenses/by/3.0/). 